\title{
Capsule Commentary on Howell et al., Incarceration History and Uncontrolled Blood Pressure in a Multi-Site Cohort
}

\author{
Aaron D. Fox, MD MS \\ Albert Einstein College of Medicine, Montefiore Medical Center, Bronx, NY, USA.
}

J Gen Intern Med 31(12): 1509

DOI: $10.1007 / \mathrm{s} 11606-016-3872-2$

(c) Society of General Internal Medicine 2016

$\mathrm{U}$ sing data from a prospective observational cohort study, Howell et al. investigated whether a history of incarceration was associated with uncontrolled blood pressure among HIV-positive and -negative patients with hypertension receiving care within the VA system. ${ }^{1}$ Participants completed surveys that included self-reporting of incarceration history, which the authors categorized as recent (within the last year), past (incarceration not within the last year), or none. Uncontrolled BP was defined as two elevated BP measurements in the 12 months following survey administration. Participants with recent incarceration had increased odds of uncontrolled blood pressure compared to those without prior incarceration, after adjusting for sociodemographic differences and cardiovascular risk factors.

These novel findings expand an emerging body of literature on the health risks associated with incarceration. In the 2 weeks following release from incarceration, the mortality rate among former prisoners is 12-fold greater than that of the general population. ${ }^{2}$ Much of this increased risk is attributable to drug overdose, but risk of cardiovascular death is also elevated and deserves additional scrutiny. ${ }^{2,3}$ This study defined recent incarceration more broadly than the immediate post-release period, and suggests that increased cardiovascular risk may persist via uncontrolled blood pressure. Study participants were already engaged in medical care, but multiple barriers to care exist following release from incarceration ${ }^{4}$; therefore, studies are needed in populations not yet engaged in care. Additional studies will also be necessary to confirm these findings and to determine a mechanism linking recent incarceration to uncontrolled blood pressure (e.g., increased stress, influence of diet and/or exercise).

The strengths of the study include an adjustment for both cardiovascular risk factors (BMI, smoking, etc.) and medical utilization (primary care engagement and anti-hypertensive medication adherence), a large multi-site cohort, and sensitivity analyses with consistent findings. However, there were some weakness: differences between groups in mean diastolic BP were small (and non-significant for systolic BP), measures of substance use were relatively imprecise, and other potential unmeasured confounders may exist.

This data does not allow causal inference about the role of recent incarceration in blood pressure control, but it should alert general internists to a potential role for incarceration in exacerbating health disparities.

Corresponding Author: Aaron D. Fox, MD MS; Albert Einstein College of Medicine, Montefiore Medical Center, Bronx, NY, USA (e-mail: adfox@montefiore.org).

\section{Compliance with Ethical Standards:}

Conflict of Interest: The author has no conflict of interest with this article.

\section{REFERENCES}

1. Howell BA, Long JB, Edelman EJ, McGinnis KA, Rimland D, Fiellin DA, Justice AC, Wang EA. Incarceration history and uncontrolled blood pressure in a multi-site cohort. J Gen Intern Med. 2016. doi:10.1007/ s11606-016-3857-1.

2. Binswanger IA, Stern MF, Deyo RA, Heagerty PJ, Cheadle A, Elmore JG, Koepsell TD. Release from prison-a high risk of death for former inmates. N Engl J Med. 2007;356(2):157-165.

3. Donahue J. Coronary artery disease in offending populations: incarceration as a risk factor and point of intervention. $J$ Correct Health Care. 2014;20(4):302-312.

4. Binswanger IA, Nowels C, Corsi KF, et al. "From the prison door right to the sidewalk, everything went downhill," a qualitative study of the health experiences of recently released inmates. Int $J$ Law Psychiatry. 2011;34(4):249-55.

Published online September 30, 2016 